\section{Journal of \\ Epilepsy and \\ Clinical \\ Neurophysiology}

J Epilepsy Clin Neurophysiol 2006; 12(2):79-88

\title{
Epilepsia e Saúde Mental na Infância
}

\author{
Heber de Souza Maia Filho*, Célia Regina Machado da Costa**, Marleide da Mota Gomes*** \\ Instituto de Psiquiatria - IPUB/UFRJ
}

\begin{abstract}
RESUMO
Objetivo: Revisar critica e sistematicamente a literatura atual sobre as comorbidades neuropsiquiátricas em crianças e adolescentes com epilepsia e seu impacto na qualidade de vida destes pacientes. Metodologia: Revisão da literatura em revistas indexadas através do MEDLINE, período de janeiro de 2000 a dezembro de 2005. Foram selecionados, a partir dos resumos, os estudos originais (epidemiológicos, diagnósticos e terapêuticos), as meta-análises, revisões sistemáticas e revisões em língua portuguesa e inglesa. Resultados: As taxas de transtornos mentais nos pacientes estudados são maiores do que em crianças normais ou mesmo com outras doenças crônicas. Uma vez que a epilepsia é uma síndrome heterogênea, múltiplos fatores podem determinar o desenvolvimento de psicopatologia. $\mathrm{O}$ artigo revisa as relações entre epilepsia e retardo mental, transtornos abrangentes, transtornos da linguagem, aprendizagem e TDAH, transtornos ansiosos e do humor, psicoses, além de aspectos diagnósticos e terapêuticos e prognóstico. Conclusões: $O$ tratamento moderno da criança com epilepsia ultrapassa o simples controle das crises epilépticas, devendo ser abrangidos o adequado diagnóstico e tratamento das comorbidades psiquiátricas a fim de melhorar a sua qualidade de vida.
\end{abstract}

Unitermos: epilepsia, criança, adolescente, comorbidade, saúde mental, qualidade de vida.

\begin{abstract}
Epilepsy and child mental health

Objective: To review the current literature about neuropsychiatry co-morbidities of children and adolescents with epilepsy and the impact of these problems in the quality of life of these patients. Methodology: Literature review in journals indexed through MEDLINE, from January 2000 to December 2005. We selected from the abstracts, original studies (epidemiology, diagnoses and therapeutics), meta-analyses, and reviews in Portuguese and English. Results: The rates of mental disorders in the patients studied are higher than in normal children or even in children with other chronic diseases. Once the epilepsy is a heterogeneous syndrome, multiple factors can determine the development of psychopathology. This paper reviews the relationships among epilepsy and mental retardation, pervasive disorder, language and learning disorders and attention deficit disorder, anxiety and mood disorders, psychoses, as well as diagnoses, therapeutic and prognostic aspects. Conclusions: The modern treatment of children with epilepsy goes beyond the simple control of the epileptic seizures and should include the appropriate diagnosis and treatment of the psychiatric co-morbidities in order to improve their life quality.
\end{abstract}

Key words: epilepsy, children, adolescents, comorbidity, mental health, quality of life.

\footnotetext{
* Doutorando (bolsista CNPq) do Programa de Pós-Graduação em Saúde Mental (PROPSAM) do Instituto de Psiquiatria - IPUB/UFRJ. Professor Auxiliar do Departamento de Medicina Materno-Infantil da Faculdade de Medicina de Petrópolis.

** Estagiária (Mestrado) do PROPSAM do IPUB/UFRJ.

*** Coordenadora do Programa de Epilepsia do Instituto de Neurologia Deolindo Couto e da linha de pesquisa sobre Epilepsia do PROPSAM. Professora Adjunta da Faculdade de Medicina da UFRJ.

Received Jan. 24, 2006; accepted May 02, 2006.
} 


\section{INTRODUÇÃO}

As comorbidades psiquiátricas e comportamentais afetam aproximadamente 40 a 50\% das crianças e adolescentes com epilepsia $(\mathrm{CCE})^{(52)}$. Algumas comorbidades são compartilhadas por adultos e crianças (depressão e ansiedade), enquanto outras são específicas da infância (autismo, transtornos da aprendizagem e transtorno do déficit de atenção e hiperatividade - TDAH) ${ }^{(50)}$. Alguns problemas são mais especificamente associados à epilepsia, tais como o transtorno do déficit de atenção e hiperatividade (TDAH), distúrbios do pensamento e problemas sociais ${ }^{(52)}$. Mesmo as síndromes epilépticas classicamente consideradas benignas demonstram em estudos de follow up uma taxa significativa de alterações comportamentais e cognitivas, como a epilepsia mioclônica benigna da infância ${ }^{(35)}$ e epilepsia rolândica ${ }^{(37)}$.

Noeker et al. ${ }^{(40)}$, propõem um modelo heurístico interessante para as interfaces entre a epilepsia e a disfunção mental. Segundo esse modelo, o surgimento de disfunção mental na criança com epilepsia poderia ser entendido em três níveis: causas de base, fatores mediadores/moderadores e prognóstico de saúde mental. As causas patogenéticas envolvem a doença de base do sistema nervoso central e as características da síndrome epiléptica (tipo de crise, freqüência e duração das crises e duração da epilepsia). Os fatores mediadores e moderadores envolvem os efeitos terapêuticos e efeitos colaterais das drogas antiepilépticas (DAE); os processos psicológicos de adaptação ao diagnóstico, tratamento e repercussões sociais da epilepsia; o nível de desenvolvimento neurocognitivo e maturidade comportamental; riscos contextuais e fatores de proteção do ambiente familiar e social. O prognóstico de saúde mental engloba três principais aspectos: a psicopatologia, o comprometimento da qualidade de vida (alterações subclínicas) e o comprometimento da capacidade de aprendizagem. O papel exato das crises epilépticas (CE) no desenvolvimento da psicopatologia é difícil de ser avaliado em razão dos fatores confusionais trazidos pela doença neurológica de base e DAE utilizadas, bem como pelas respostas familiares ao problema ${ }^{(6)}$. Mesmo assim, alguns estudos propõem um papel mais relevante das CE na gênese dos problemas mentais e comportamentais das crianças com epilepsia, incluindo o papel de alterações epileptogênicas interictais e o comprometimento cognitivo transitório ${ }^{(6)}$. Um fator a mais a ser considerado nesta interrelação é a maior incidência de eventos não epilépticos psicogênicos em portadores de epilepsia e comorbidades psiquiátricas, particularmente naqueles com depressão e transtornos ansiosos (transtorno obsessivo-compulsivo e estresse pós-traumático) ${ }^{(47)}$. Assim, a epilepsia, por suas interações com diversas doenças psiquiátricas, pode servir como um modelo para o entendimento das bases neurológicas da doença mental. Em face da complexidade e prevalência das questões apontadas resolvemos revisar critica e sistematicamente a literatura atual sobre as comorbidades neuropsiquiátricas em crianças e adolescentes com epilepsia e seu impacto na qualidade de vida.

\section{METODOLOGIA}

Revisão da literatura nacional e internacional em revistas indexadas através do MEDLINE, período de janeiro de 2000 a dezembro de 2005, utilizando o MeSH Term "epilepsy" associado a cada um dos MeSH Terms seguintes isoladamente: "mental health", "comorbidity", "psychopathology", "child psychiatry", adolescent psychiatry", 'anxiety disorders", "depressive disorder", "autism", "conduct disorder", "obsessive-compulsive disorder", "attention deficit and hyperactivity disorder - ADHD", "learning disorders", "dyslexia”, "dyscalculia”, "language disorders" e "transient cognitive impairment" (palavras no título e resumo, restringindo para faixa etária pediátrica e pesquisas em seres humanos); "behavior" e "mental retardation" (palavras apenas no título, com as mesmas restrições). Foram selecionados, a partir dos resumos, os estudos originais (epidemiológicos, diagnósticos e terapêuticos), as meta-análises, revisões sistemáticas e revisões em língua portuguesa e inglesa (123 artigos). Alguns artigos de interesse das referências não abrangidos pela ferramenta de busca foram incluídos. Poucos estudos com mais de dez anos foram selecionados por discutirem algum aspecto original que não tenha sido referido ou contestado posteriormente. $O$ final total foi de 62 artigos.

\section{RESULTADOS}

\section{Epidemiologia}

As taxas de transtorno mental em CCE são maiores do que em crianças normais ou mesmo com outras doenças crônicas (Tabela 1). Davies et al. ${ }^{(23)}$ realizaram estudo populacional na Inglaterra, encontrando uma taxa de 37\% de transtornos psiquiátricos em CCE, comparativamente a $11 \%$ em crianças diabéticas e $9 \%$ na população geral. Rodenburg et al. ${ }^{(56)}$ realizaram meta-análise de 46 estudos, totalizando 2434 CCE. As diferenças encontradas nas freqüências de psicopatologia foram maiores quando comparadas a crianças saudáveis do que com aquelas com outras doenças, demonstrando que parte dos problemas encontrados pode ser atribuído à cronicidade da doença. Os problemas mais específicos foram o distúrbio de atenção, alterações do pensamento e problemas sociais. Keene et al.(31) encontraram uma prevalência de psicopatologia geral de 15,9\%. Poucos estudos foram conduzidos em países em desenvolvimento, embora $90 \%$ das crianças epilépticas vivam nestes países ${ }^{(1,7,21,53,61)}$. $\bigcirc$ único estudo nacional ${ }^{(61)}$ encontra uma alta taxa de comorbidade psiquiátrica (70,5\%). Trata-se de uma amostra específica de um ambulatório terciário de psiquiatria em epilepsia, com alta taxa de epilepsias refratárias e QI na faixa médio inferior $(80 \pm 19,4)$. 
Tabela 1. Estudos de prevalência/incidência de psicopatologia em crianças e adolescentes com epilepsia

Continua

\begin{tabular}{|c|c|c|c|c|c|c|c|c|c|c|c|c|c|}
\hline Estudo & Local & Tipo & Amostra & Idade & Instrumentos & Total & R.M. & Autismo & TDAH & $\begin{array}{c}\text { Depres- } \\
\text { são }\end{array}$ & Conduta & Outros & Fatores preditivos \\
\hline $\begin{array}{l}\text { Adewuya et al., } \\
2005\end{array}$ & Nigéria & Transversal & $102 \mathrm{CCE}$ & $12-18$ anos & $\begin{array}{l}\text { DISC-IV, GHQ, } \\
\text { FILEC, FIRM, } \\
\text { CATIS }\end{array}$ & - & - & - & - & $28,43 \%$ & - & - & $\begin{array}{l}\text { Ansiedade: percepcão } \\
\text { do estigma, frequêência } \\
\text { crises, número DAE, } \\
\text { psicopatologia pais, } \\
\text { estressores familiares e } \\
\text { percepção parental de } \\
\text { estigma. } \\
\text { Depressão: percepção } \\
\text { do estigma, frequiência } \\
\text { crises, número DAE, } \\
\text { psicopatologia pais e } \\
\text { estressores familiares }\end{array}$ \\
\hline $\begin{array}{l}\text { Austin et al., } \\
2000\end{array}$ & EUA & $\begin{array}{l}\text { Longitudinal } \\
\text { (4 anos) }\end{array}$ & $\begin{array}{l}115 \mathrm{CCE} \\
105 \mathrm{ASM}\end{array}$ & $8-13$ anos & CBCL & - & - & - & - & - & - & $\begin{array}{l}\text { O grupo como um } \\
\text { todo teve melhora } \\
\text { na pontuação total, } \\
\text { comportamento } \\
\text { internalizante e } \\
\text { externalizante, à } \\
\text { exceção de } \\
\text { meninas } \\
\text { adolescentes }\end{array}$ & - \\
\hline $\begin{array}{l}\text { Baki et al., } \\
2004\end{array}$ & Turquia & $\begin{array}{l}\text { Transversal, } \\
\text { caso-controle }\end{array}$ & $\begin{array}{c}\text { Clínica } \\
35 \mathrm{CCE} \\
35 \mathrm{~N} \\
\text { Respectivos } \\
\text { pais }\end{array}$ & 7-19 anos & $\begin{array}{l}\text { IDB, STAI, IDCK } \\
\text { STAIc }\end{array}$ & - & - & - & - & $\begin{array}{l}9 \%(\mathrm{CCE}) \\
\mathrm{X} 5 \%(\mathrm{~N})\end{array}$ & - & $\begin{array}{l}\text { Mãe de CCE não } \\
\text { têm maior } \\
\text { prevalência de } \\
\text { depressão e } \\
\text { ansiedade do que } \\
\text { mães de N }\end{array}$ & - \\
\hline $\begin{array}{l}\text { Caplan et al., } \\
2005\end{array}$ & EUA & Transversal & $\begin{array}{c}\text { Clínica } \\
100 \mathrm{CPC} \\
71 \mathrm{CA} \\
93 \mathrm{~N}\end{array}$ & 5-16 anos & $\begin{array}{l}\text { K-SADS, CBCL } \\
\text { CDI, MASC, } \\
\text { WISC-R, TOLD }\end{array}$ & - & - & - & - & $6,4 \%$ & - & $\begin{array}{l}20 \% \text { ideação } \\
\text { suicida; } \\
30 \% \text { receberam } \\
\text { atendimento de } \\
\text { saúde mental }\end{array}$ & $\begin{array}{l}\text { Idade, QI verbal, } \\
\text { problemas escolares, } \\
\text { tipo de crise } \\
\text { (ansiedade) e duração } \\
\text { da doença (ideação } \\
\text { suicida). }\end{array}$ \\
\hline $\begin{array}{l}\text { Datta et al., } \\
2005\end{array}$ & Índia & Transversal & $132 \mathrm{CCE}$ & $\begin{array}{c}11,03 \pm \\
3,65 \text { anos }\end{array}$ & CBCL & $53,8 \%$ & - & - & - & - & - & - & $\begin{array}{l}\text { Maior nível } \\
\text { socioeconômico, } \\
\text { politerapia e tempo de } \\
\text { doença (aumento da } \\
\text { psicopatologia) }\end{array}$ \\
\hline $\begin{array}{l}\text { Davies et al., } \\
2003\end{array}$ & Inglaterra & Transversal & $\begin{array}{c}\text { Populacional } \\
63 \mathrm{CCE} \\
47 \mathrm{DM} \\
10202 \mathrm{~N}\end{array}$ & 5-15 anos & $\begin{array}{c}\text { DWBA + } \\
\text { entrevista clínica }\end{array}$ & $\begin{array}{c}37 \% \mathrm{CCE} \\
11 \% \mathrm{DM} \\
9 \% \mathrm{~N}\end{array}$ & - & $16 \%$ & $12 \%$ & - & $30,7 \%$ & $\begin{array}{l}\text { Transtornos } \\
\text { emocionais (não } \\
\text { especificados) } \\
32,7 \%\end{array}$ & - \\
\hline
\end{tabular}

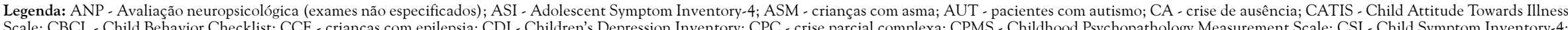

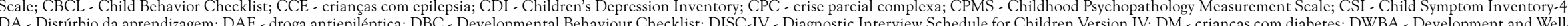

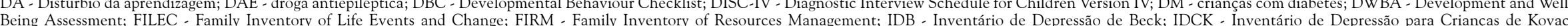

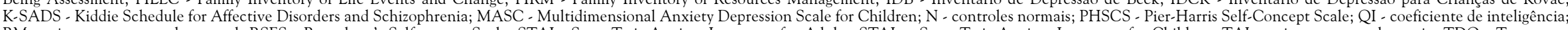

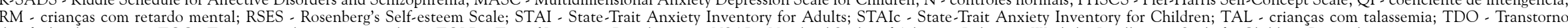
desafiador de oposição; TOC - Transtorno obsessivo compulsivo; TOLD - Test of Language Development; VSAB - Vineland Scales of Adaptive Behavior; Wechsler Intelligence Scale for Children-Revised 
Tabela 1. Estudos de prevalência/incidência de psicopatologia em crianças e adolescentes com epilepsia

Continuação

\begin{tabular}{|c|c|c|c|c|c|c|c|c|c|c|c|c|c|}
\hline Estudo & Local & Tipo & Amostra & Idade & Instrumentos & Total & R.M. & Autismo & TDAH & $\begin{array}{c}\text { Depres- } \\
\text { são }\end{array}$ & Conduta & Outros & Fatores preditivos \\
\hline $\begin{array}{l}\text { Dunn et al., } \\
2003\end{array}$ & EUA & Transversal & $175 \mathrm{CCE}$ & 9-14 anos & $\begin{array}{l}\text { CBCL, CSI } \\
\text { ASI }\end{array}$ & - & - & - & \multicolumn{2}{|c|}{$\begin{array}{c}11.4 \% \\
\text { Combinado } \\
24 \% \\
\text { Desatento } \\
2,3 \% \\
\text { Hiperativo }\end{array}$} & - & - & $\begin{array}{l}\text { Sexo, tipo de crise e } \\
\text { localização do foco } \\
\text { epiléptico não tiveram } \\
\text { correlação diagnóstica }\end{array}$ \\
\hline Transversal & $\begin{array}{l}\text { Clínica } \\
175 \text { CCE }\end{array}$ & 6-18 anos & CBCL & \multicolumn{2}{|c|}{$\begin{array}{c}15,9 \% \text { total } \\
12,7 \% \\
\text { internalizante } \\
11,4 \% \\
\text { externalizante }\end{array}$} & - & - & - & $\begin{array}{l}\mathrm{c} \\
\mathrm{n} \\
\mathrm{t} \\
\mathrm{p} \\
\mathrm{a} \\
\mathrm{u} \\
\mathrm{s}\end{array}$ & $\begin{array}{l}\text { Crianças com } \\
\text { maior pontuaçãc } \\
\text { tiveram mais } \\
\text { problemas de } \\
\text { aprendizado e } \\
\text { usaram mais os } \\
\text { serviços de saúd }\end{array}$ & - & $\begin{array}{l}\text { Crianças com } \\
\text { maior pontuação } \\
\text { tiveram mais } \\
\text { problemas de } \\
\text { aprendizado e } \\
\text { usaram mais os } \\
\text { serviços de saúde }\end{array}$ & - \\
\hline Coorte & \multicolumn{2}{|l|}{$\begin{array}{l}\text { Populacional } \\
392 \text { RM }\end{array}$} & DBC & \multicolumn{2}{|c|}{$\begin{array}{c}43,5 \% \mathrm{CCE} \\
41,1 \% \mathrm{~N}\end{array}$} & - & - & - & - & - & - & - & $\begin{array}{l}\text { O RM não confere } \\
\text { aumento de } \\
\text { psicopatologia }\end{array}$ \\
\hline $\begin{array}{l}\text { Ott et al., } \\
2001\end{array}$ & EUA & Transversal & $\begin{array}{c}\text { Clínica } \\
48 \mathrm{CPC} \\
39 \mathrm{CA} \\
59 \mathrm{~N}\end{array}$ & 5-16 anos & K-SADS, CBCL & $\begin{array}{c}26 \%(\mathrm{CBCL}) \\
51 \%(\mathrm{~K}-\mathrm{SADS})\end{array}$ & - & - & - & - & - & $\begin{array}{l}\text { O K-SADS } \\
\text { identifica um } \\
\text { maior número de } \\
\text { crianças com } \\
\text { problemas mentais }\end{array}$ & QI total \\
\hline $\begin{array}{l}\text { Ott et al., } \\
2003\end{array}$ & EUA & Transversal & $\begin{array}{c}\text { Clínica } \\
114 \mathrm{CPC} / \mathrm{CA}\end{array}$ & 5-16 anos & $\begin{array}{l}\text { K-SADS, CBCL } \\
\text { TOLD, WISC-R }\end{array}$ & $\begin{array}{c}60,5 \% \\
\text { CBCL: } 53 \% \\
\text { internalizante; } \\
49,2 \% \\
\text { externalizante }\end{array}$ & $\begin{array}{c}\text { Realizada } \\
\text { avaliação } \\
\text { QI, mas } \\
\text { não } \\
\text { descrita }\end{array}$ & - & - & - & $23 \%$ & $\begin{array}{l}>60 \% \text { não } \\
\text { recebiam nenhum } \\
\text { atendimento de } \\
\text { saúde mental }\end{array}$ & $\begin{array}{l}\text { Fatores relacionados à } \\
\text { ausência de } \\
\text { atendimento } \\
\text { psiquiátrico): } \\
\text { Menos idade, menor } \\
\text { nível educacional dos } \\
\text { pais, menor número de } \\
\text { DAE, maior QI verbal, } \\
\text { CAE, diagnóstico } \\
\text { psiquiátrico único }\end{array}$ \\
\hline $\begin{array}{l}\text { Pradhan et al., } \\
2003\end{array}$ & Î́ndia & Transversal & $\begin{array}{l}\text { Clínica } \\
\text { 30 CCE } \\
\text { 30 TAL }\end{array}$ & 8-12 anos & CPMS, RSES & $\begin{array}{c}\text { CCE } 26,06 \\
\text { TAL } 28,56 \%\end{array}$ & - & - & - & - & - & $\begin{array}{l}\text { A auto-estima foi } \\
\text { moderadamente } \\
\text { comprometida nos } \\
\text { dois grupos }\end{array}$ & - \\
\hline $\begin{array}{l}\text { Thome-Souza } \\
\text { et al., 2004 }\end{array}$ & Brasil & Tranversal & $\begin{array}{l}\text { Clínica } \\
78 \text { CCE }\end{array}$ & 4-18 anos & $\begin{array}{c}\text { Avaliação } \\
\text { psiquiátrica } \\
\text { K-SADS, ANP }\end{array}$ & $70,5 \%$ & $\begin{array}{l}\text { QI médio } \\
\text { da amostra } \\
\text { foi baixo: } \\
80 \pm 19,4\end{array}$ & $9,1 \%$ & $29,1 \%$ & $36,4 \%$ & $\begin{array}{l}16,5 \% \\
\text { (TDO) }\end{array}$ & $\begin{array}{l}\text { 9\% apresentavam } \\
\text { outros } \\
\text { diagnósticos } \\
\text { (TOC, ansiedade, } \\
\text { DA, TBH), não } \\
\text { sendo especificada } \\
\text { a prevalência } \\
\text { isolada de cada um. }\end{array}$ & - \\
\hline
\end{tabular}

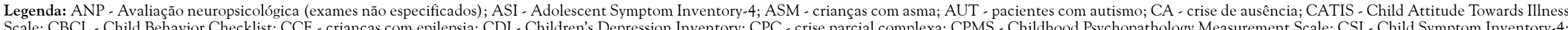
Scale; CBCL - Child Behavior Checklist; CCE - crianças com epilepsia; CDI - Children's Depression Inventory; CPC - crise parcial complexa; CPMS - Childhood Psychopathology Measurement Scale; CSI - Child Symptom Inventory-4 Being Assessment; FILEC - Family Inventory of Life Events and Change; FIRM - Family Inventory of Resources Management; IDB - Inventário de Depressão de Beck; IDCK - Inventário de Depressão para Crianças de Kovac,

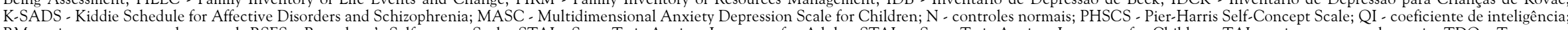

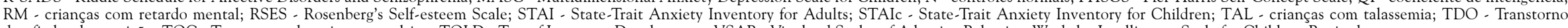
desafiador de oposição; TOC - Transtorno obsessivo compulsivo; TOLD - Test of Language Development; VSAB - Vineland Scales of Adaptive Behavior; Wechsler Intelligence Scale for Children-Revised. 


\section{Fatores de Risco}

Uma vez que a epilepsia é uma síndrome heterogênea, múltiplos fatores podem determinar o desenvolvimento de psicopatologia ${ }^{(24)}$. Caplan et al. ${ }^{(18)}$ encontraram associação de diagnóstico de transtorno de ansiedade com a idade, baixo QI verbal, problemas escolares e tipo de crise. Encontraram correlação de ideação suicida com a duração da epilepsia. O baixo nível intelectual é um fator associado ao agravo dos problemas de saúde mental em vários estudos ${ }^{(13)}$. Adewuya et al. ${ }^{(1)}$ encontraram como fatores preditivos de ansiedade e depressão em adolescentes epilépticos na Nigéria os seguintes fatores: CE não controladas, politerapia e estigma percebido. Datta et al.(21) encontraram como fatores de risco principais para psicopatologia em CCE: politerapia, maior nível socioeconômico em países em desenvolvimento e maior tempo de doença. Comparativamente a grupos controles, famílias com uma CCE têm maior comprometimento em diversos aspectos, tais como a baixa qualidade da relação paisfilhos, depressão materna e funcionamento familiar. Rodenburg et al. sugerem que a psicopatologia da CCE está mais associada ao controle psicológico dos pais, atributos dados pelos pais à epilepsia e satisfação familiar com os relacionamentos intrafamiliares ${ }^{(55)}$. As mães costumam envolver-se emocionalmente de forma intensa com seus filhos com epilepsia, bem como demonstram maior hostilidade e atitude crítica. Esses últimos sentimentos associam-se a maior comportamento anti-social e hiperativo neles ${ }^{(29)}$. Brent et al. ${ }^{(11)}$ notaram uma maior incidência de depressão em crianças em uso de fenobarbital na presença de história familiar positiva para transtorno depressivo em parentes de primeiro grau. A depressão persistiu naqueles pacientes que mantiveram o tratamento com a droga e remitiu naqueles que trocaram de medicação ${ }^{(12)}$. Os resultados de estudos sobre as relações entre gênero e psicopatologia na epilepsia infantil são inconsistentes. Austin et al. ${ }^{(5)}$ desenvolveram o estudo mais recente sobre o assunto, demonstrando que meninas adolescentes com epilepsia de difícil controle apresentam uma maior prevalência de problemas comportamentais internalizantes (ansiedade, inibição, sintomas depressivos e dificuldade de atenção) e externalizantes (comportamento agressivo e anti-social), quando comparadas com os meninos epilépticos e adolescentes com asma leve e grave de ambos os sexos. O grau de comprometimento psicopatológico aumentou ao longo do tempo (seguimento de 4 anos). Cortesi et al. ${ }^{(19)}$ demonstraram que a idade de início das CE, as anormalidades do eletroencefalograma (EEG), a duração da doença, a freqüência das crises e os problemas comportamentais foram mais associados a problemas do sono. Becker et al. ${ }^{(8)}$ analisaram 30 CCE (57\% com epilepsias generalizadas), demonstrando $80 \%$ de alterações do sono (apnéias e hipopnéias e alterações na arquitetura do sono no registro polissonográfico), bem como sonolência diurna, o que foi significativamente associado a sintomas de desatenção e hiperatividade, comportamento opositivo e sintomas depressivos.

\section{Retardo Mental}

Diversos estudos demonstram relação entre o baixo QI e psicopatologia aumentada nas $\operatorname{CCE}^{(13,15,16,18)}$. Os estudos de Caplan et al. ${ }^{(15,16,18)}$ não são focados diretamente na questão do baixo QI, mas demonstram sua associação com maior psicopatologia e alterações do pensamento formal. Buelow et al. ${ }^{(13)}$ analisaram 164 CCE, subdividindo-os em três grupos de acordo com o nível intelectual (QI baixo, médio ou acima da média). As crianças com QI mais baixo apresentaram maior índice de psicopatologia, mais baixo autoconceito e maior prevalência de depressão. Os problemas com autoconceito foram maiores no sexo feminino. Steffenburg et al. ${ }^{(59)}$ encontraram 59\% de uma amostra populacional de 98 crianças com retardo mental e epilepsia tendo pelo menos um diagnóstico psiquiátrico. Trinta e três por cento dessas crianças não puderam ter um diagnóstico psiquiátrico preciso em razão de profundo retardo mental. Lewis et al. ${ }^{(34)}$ fizeram análise diversa, procurando concluir se, em crianças com retardo mental, a presença de epilepsia aumenta a taxa de psicopatologia. Diferente dos estudos de Caplan e Buelow (amostras clínicas), este estudo é populacional. Os autores não encontraram diferença na intensidade de psicopatologia em crianças com retardo mental com e sem epilepsia. O retardo mental, portanto, seria um fator independente que confere maior chance de problemas de saúde mental para crianças com epilepsia. Crianças com síndromes específicas associadas ao retardo mental têm padrões mais bem definidos de psicopatologia ${ }^{(17)}$. Um exemplo é a associação entre esclerose tuberosa, Síndrome de West e transtorno autista ${ }^{(10)}$.

\section{Transtornos Abrangentes do Desenvolvimento}

A associação entre epilepsia e autismo já se encontra no estudo original de Leo Kanner quando, em 1944, descreve um de seus pacientes como sofrendo de epilepsia (apud 39). A prevalência da epilepsia no autismo varia entre $8 \mathrm{e}$ $42 \%{ }^{(14)}$. Essa maior prevalência parece ser mais associada a comorbidades e doença neurológica de base do que ao autismo per se. Mais particularmente, Pavone et al..$^{(50)}$ encontraram uma prevalência de epilepsia no autismo primário de 7,4\% e de 55\% no autismo secundário. Steffenburg et al. ${ }^{(60)}$ encontraram uma maior prevalência de epilepsia sintomática (causas perinatais), com crises parciais em crianças com transtorno autista. $\mathrm{O}$ início das crises nesse grupo é mais tardio. Apesar da natureza sintomática, não foi encontrada uma prevalência aumentada de paralisia cerebral e deficiências sensoriais nessa amos- 
tra. Gabis et al. ${ }^{(23)}$ analisaram retrospectivamente uma amostra de 56 crianças com transtornos do espectro autista, diagnosticando epilepsia em $28,6 \%$ e encontrando anormalidades eletroencefalográficas em 30\% das mesmas. As crianças tinham sido encaminhadas para EEG por diversos motivos, nem sempre com queixa específica de crise epiléptica. Não foi analisada a associação entre o diagnóstico de epilepsia e as alterações do EEG. Canitano et al.(14) não encontraram associação entre anormalidades do EEG e história de regressão comportamental e de linguagem no período de 2 a 3 anos de idade em 77 crianças com autismo, associado ou não à epilepsia. Hrdlicka et al. ${ }^{(30)}$ encontraram resultados semelhantes em relação aos aspectos do EEG, mas notaram uma associação significativa entre o diagnóstico de epilepsia e a regressão autista. Pacientes com psicose desintegrativa têm uma chance maior de desenvolver epilepsia do que portadores de autismo infantil. Esse parece ser um grupo distinto de crianças com transtorno abrangente do desenvolvimento, com possível neuropatologia de base contribuindo para as diversas manifestações, incluindo a epilepsia ${ }^{(38)}$.

\section{Transtornos Psicóticos}

Sintomas psicóticos ocorrem com maior freqüência em crianças com crises parciais complexas ${ }^{(16)}$. Caplan et al. ${ }^{(15)}$ analisaram CCE quanto ao curso de pensamento formal (lógica e associação), encontrando uma maior freqüência de pensamento ilógico naquelas com crises parciais complexas que apresentavam disfunção cognitiva global e sintomas psicóticos.

\section{Distúrbios da Linguagem e Aprendizagem}

A epilepsia e seu tratamento podem se associar a alterações no desenvolvimento da linguagem de várias maneiras. As crises podem ser um sintoma de lesão em áreas essenciais para a função lingüística e as conseqüências das CE e das DAE podem influenciar na aprendizagem ${ }^{(27)}$. Uma condição que bem representa as relações entre a epilepsia e a linguagem é a Síndrome de Landau Klefner. A síndrome é caracterizada por uma agnosia auditivo-verbal adquirida (alterações na linguagem receptiva por disfunção na decodificação fonológica) associada a crises epilépticas e/ou alterações eletroencefalográficas durante o registro de sono ${ }^{(27)}$. Parkinson et al. ${ }^{(49)}$, avaliando um grupo de 109 crianças epilépticas, demonstraram a associação entre CE focais (simples ou complexas) e distúrbios lingüísticos. A amostra era composta principalmente de epilepsias sintomáticas de difícil tratamento (Síndrome de West, LennoxGastaut, epilepsias de lobo frontal e temporal), demonstrando um substrato orgânico em comum. Embora as crianças com epilepsia benigna da infância com pontas centro-temporais (epilepsia rolândica) tenham bom prognóstico, alguns estudos sugerem a existência de distúrbios de linguagem associados à disfunção interictal nas áreas perisilvianas de linguagem ${ }^{(37)}$. $O$ prognóstico a longo prazo é associado a uma diversidade de déficits neuropsicológicos ${ }^{(19)}$. Papavasiliou et al. ${ }^{(48)}$ acompanharam um grupo de 32 CCE rolândica típica, mas com difícil controle, demonstrando pior desempenho do que controles em relação à soletração, leitura em voz alta e compreensão de texto lido, características típicas de dislexia e mau desempenho em testes cognitivos não verbais. Entretanto, o grupo apresentava nível cognitivo geral mais baixo e alterações no desenvolvimento pré-existente, lançando dúvidas sobre uma associação entre a epilepsia rolândica e os distúrbios da aprendizagem. Monjauze et al. ${ }^{(37)}$ demonstraram alterações na gramática expressiva e habilidades de leitura, bem como déficit de linguagem em 56\% das crianças de sua amostra com epilepsia rolândica. Crianças com epilepsia encontram-se em risco de fracasso escolar. Embora esse risco seja maior para CCE sintomática (associado a comprometimento cognitivo global ou específico), esse fato também é verdadeiro para CCE idiopática. Atribui-se tal dificuldade a alterações de memória, atenção, linguagem $^{(63)}$, bem como a influência das DAE e comorbidades comportamentais. Oostrom et al. ${ }^{(44)}$ acompanharam 51 CCE idiopática ao longo de um período de 12 meses, relatando que $51 \%$ das mesmas necessitaram de apoio educacional especializado, em contraste com $27 \%$ de controles normais. As CCE apresentaram uma incidência maior de dificuldades comportamentais, problemas com a atenção, tempo de reação a estímulos e capacidade de aprendizagem. Os autores concluíram que as dificuldades cognitivas, bem como comportamentais já são perceptíveis nos primeiros estágios da doença. Aldenkamp et al. ${ }^{(4)}$, analisando um grupo de CCE e excluindo diagnósticos comórbidos (TDAH, retardo mental e dislexia) demonstraram que um quadro de crises difíceis de detectar (sintomas representando características comportamentais como lapsos de atenção e EEG com alterações específicas) pode representar uma epilepsia não controlada podendo levar a declínio escolar. A discalculia é um distúrbio neurológico específico da habilidade aritmética, ocorrendo em 5 a $6 \%$ dos escolares e sendo mais comum em uma série de distúrbios neurológicos, dentre eles a epilepsia ${ }^{(58)}$. A existência de um transtorno cognitivo transitório associado a descargas elétricas anômalas no EEG é um fenômeno recentemente discutido e provavelmente raro. Deve ser diferenciado do estado pós-ictal, das alterações do EEG assintomáticas ou típicas de determinada síndrome, presentes no EEG interictal, bem como das crises parciais complexas com sintomatologia discreta e fugaz ${ }^{(4)}$.

\section{Transtorno do Déficit de Atenção e Hiperatividade (TDAH)}

Diversos estudos demonstram a associação entre epilepsia e TDAH, através de questionários e escalas comportamentais e testes neuropsicológicos ${ }^{(57)}$. A difi- 
culdade de atenção nas CCE tem natureza multifatorial, podendo representar crises subclínicas, distúrbio de aprendizagem não diagnosticado, distúrbios do sono, efeito colateral de DAE ou TDAH propriamente dito ${ }^{(57)}$. A maioria dos estudos relata prevalência entre 8 a $77 \%$ de TDAH nas epilepsias, sendo que na população geral a prevalência do distúrbio é em torno de 5\%. Dunn et al. ${ }^{(23)}$ relataram $11,4 \%$ do subtipo combinado, $24 \%$ do subtipo desatento e 2,3\% do subtipo hiperativo, diferentemente da população geral, onde o subtipo combinado é o mais prevalente. $\mathrm{O}$ estudo foi realizado a partir de uma amostra clínica, com o uso de três escalas diagnósticas (Child Behavior Checklist, Child Symptom Inventory-4 e Adolescent Symptom Inventory4) que não são específicas para o diagnóstico de TDAH, não tendo sido feita confirmação posterior do mesmo. Os seus resultados não foram controlados para o tratamento do TDAH que já poderia estar ocorrendo. Hesdorffer et al.(28) acompanharam uma coorte de CCE recémdiagnosticada. O diagnóstico de TDAH prévio era 2,5 vezes mais freqüente do que em controles saudáveis pareados. Esse achado ocorreu somente para a forma predominantemente desatenta do distúrbio. $\mathrm{O}$ estudo sugere antecedentes comuns a ambas as condições e não simplesmente uma relação de causalidade da epilepsia em relação ao déficit de atenção. $O$ grupo encontrou uma freqüência aumentada de anormalidade eletroencefalográficas, mas não foi possível correlacionar este achado com número aumentado de crises. Em um estudo avaliando características neuropsicológicas (bateria de testes computadorizados) de desatenção em CCE idiopática ou criptogenética, recémdiagnosticada, Oostrom et al. ${ }^{(44)}$ não encontraram diferenças entre as CCE e controles normais em relação a testes executivos e de velocidade motora. Ocorreram erros mais freqüentes em testes de flexibilidade cognitiva e atenção sustentada. Os pacientes com dificuldades escolares prévias ao início da epilepsia e aqueles cujos pais reagiram de forma menos adaptada ao diagnóstico tiveram este pior desempenho. Os autores concluíram que variáveis relacionadas à epilepsia não justificam os achados. Não há relato se houve diagnóstico clínico de TDAH, o que é diferente de mau desempenho em testes neuropsicológicos.

Algumas considerações de natureza clínica também podem ser encontradas nos estudos pesquisados. Embora o diagnóstico de TDAH em geral seja clínico, o EEG pode ser útil em distinguir entre inatenção comportamental e crises parciais complexas ou ausência típica, nos casos em que se suspeita de epilepsia ou esta foi previamente diagnosticada ${ }^{(57)}$. Quanto ao tratamento para o TDAH em CCE, outros estimulantes que não a bupropiona parecem ser seguros ${ }^{(57)}$.

\section{Transtornos Ansiosos e do Humor}

Baki et al. (7), comparando um grupo de 35 CCE com controles saudáveis encontraram uma prevalência de 12\% de depressão (contra 9\% nos controles) e 49\% de sintomas leves a moderados de ansiedade (não houve diferença em relação aos controles). Os autores também analisaram a prevalência de depressão e ansiedade nas mães das $\mathrm{CCE}$, não encontrando diferenças em relação aos controles. Não encontraram correlação de variáveis clínicas (tipo de crise, início de doença, freqüência de crises) com os sintomas estudados. Caplan et al.(18) encontraram uma chance cinco vezes maior de CCE apresentar transtornos afetivos ou ansiosos do que controles (prevalência geral de 33\%). A maioria das CCE acometidas apresentava transtorno ansioso isolado (63\%) ou comórbido com depressão ou transtorno disruptivo $(26,1 \%)$. A prevalência de ideação suicida também foi maior $(20 \%$ CCE $\times 9 \%$ controles), sendo o seu risco maior naqueles com transtorno disruptivo associado. Houve diferenças na prevalência dos transtornos de acordo com o tipo de epilepsia. As crianças com crises parciais complexas tiveram maior freqüência de depressão e depressão/ansiedade e aquelas com crise de ausência maior freqüência de ansiedade. Outras variáveis clínicas não foram significativas em diferenciar pacientes normais daqueles com algum tipo de psicopatologia. Apenas uma maior duração de doença foi associada à ideação suicida. Maior psicopatologia foi associada ao menor QI e problemas com escolaridade. Adewuya et al..$^{(1)}$ encontraram uma prevalência de 31,37\% de ansiedade e $28,43 \%$ de depressão em 102 adolescentes nigerianos com epilepsia. Os fatores associados à psicopatologia foram: maior percepção de estigma em relação à epilepsia, freqüência maior de crises, número aumentado de DAE, psicopatologia dos pais, estressores familiares e percepção parental de estigma. Não foram encontrados artigos relatando relação entre transtorno obsessivo-compulsivo (TOC) e epilepsia, exceto um relato de caso de uma criança com TOC e crises de difícil controle originadas na região anterior do giro do cíngulo direito ${ }^{(33)}$.

\section{Aspectos Psicossociais}

O autoconceito é definido como uma composição de valores, crenças e sentimentos que o indivíduo possui acerca dele próprio ao longo do tempo e que é formado a partir da percepção interna e percepção das reações dos outros. Pode ser compreendido como uma estrutura cognitiva de conhecimentos que orienta a nossa atenção para as informações que são relevantes para nós e nos ajudam na adaptação ao ambiente ${ }^{(26)}$. Meninas tiveram maior comprometimento de autoconceito ${ }^{(13)}$. Não foram encontradas referências à relação entre gênero e auto-estima. Pradhan et al.(53) estudaram a auto-estima em CCE e talassemia, achando comprometimento da mesma em ambos os grupos, o que foi associado a alto nível de psicopatologia, em especial depressão. As CCE têm padrões semelhantes a controles normais na maneira em que 
entendem e atribuem noções de culpa e vergonha. É um sentimento semelhante nestes grupos atribuir tais sentimentos à sensação de incompetência causada pela epilepsia. Tal resultado é importante, pois vergonha e culpa são freqüentes sentimentos desencadeadores de psicopato$\operatorname{logia}{ }^{(42)}$.

A reação de ajustamento à nova condição de vida (ter epilepsia) parece relacionada a um risco aumentado de reações negativas. Tal reação mostra-se comprometida naquelas crianças com encefalopatia epiléptica e epilepsias sintomáticas. Nas demais situações, o comportamento adaptativo da CCE em geral é comparável a crianças saudáveis e tende a permanecer estável ao longo do tempo ${ }^{(9)}$.

\section{Saúde Mental e Qualidade de Vida}

Embora o campo de estudo da qualidade de vida relacionada à saúde (QVRS) na epilepsia infantil esteja avançado, encontramos um único estudo analisando especificamente o impacto da comorbidade psiquiátrica na vida da CCE, mais especificamente de adolescentes ${ }^{(2)}$. Os autores encontraram comprometimento da QVRS associado a sintomas de ansiedade e depressão. Os autores concluíram que a comorbidade psiquiátrica explica mais as variações da QVRS de CCE do que as variáveis clínicas e sociodemográficas.

\section{Aspectos Diagnósticos}

Os estudos usam diferentes instrumentos diagnósticos, bem como as amostras diferem muito em relação a tamanho, faixa etária e tipo de epilepsia, o que torna os dados pouco comparáveis. O Kiddie Schedule for Affective Disorders and Schizophrenia (K-SADS) quando utilizado no diagnóstico de comorbidades psiquiátricas detecta um maior número de crianças com problemas quando comparado ao Child Behavior Checklist (CBCL) segundo Ott et al.(45). O K-SADS é um instrumento de diagnóstico psiquiátrico específico, enquanto que o CBCL gera categorias amplas de psicopatologia (problemas internalizantes e externalizantes). Devemos tomar alguns cuidados com o uso de escalas diagnósticas psiquiátricas em crianças com epilepsia, uma vez que certos descritores comportamentais podem ser confundidos com características da crise epiléptica (particularmente ausência e parcial complexa). Oostrom et al.(45) ao estudarem este problema demonstraram uma redução de $46 \%$ para $23 \%$ de psicopatologia diagnosticada pelo CBCL e de 18 para 15\% no Teacher's Report Form (TRF) quando foram suprimidos itens sujeitos à interpretação ambígua. Isso deve levar a algumas reservas na interpretação destes escores.

\section{Aspectos Terapêuticos}

Diversos estudos demonstram que as CCE não têm seus problemas psiquiátricos comórbidos adequadamente diagnosticados e tratados ${ }^{(18)}$. Os fatores associados a um menor encaminhamento para atendimento psiquiátrico são: menos idade, menor nível educacional dos pais, menor número de DAE utilizadas, maior QI verbal, epilepsia de ausência e diagnóstico psiquiátrico único ${ }^{(45)}$. Crianças com baixo QI têm maior chance de serem encaminhadas a atendimento de saúde mental. Crianças com inteligência normal têm melhor ajustamento social, ficando os problemas emocionais desconhecidos por maior tempo ${ }^{(4)}$. $\mathrm{O}$ tratamento de CCE e comorbidades psiquiátricas deve ser individualizados. O objetivo é conseguir o controle das crises e o melhor funcionamento físico, cognitivo e emocional com o menor número de medicações possível ${ }^{(51)}$. Wheless et al. ${ }^{\left({ }^{(62)}\right.}$ propõem um algoritmo de escolha de DAE que leva em consideração não somente seu efeito da síndrome epiléptica em questão, mas também sua eficácia nas comorbidades psiquiátricas presentes, estimulando uma redução da polifarmácia nestes pacientes. Pressler et al. ${ }^{(54)}$ realizaram ensaio terapêutico duplo cego placebo controlado com o uso de lamotrigina e observaram melhora dos escores em escala comportamental apenas naqueles pacientes que tiveram concomitante redução de descargas anômalas interictais no EEG. O resultado foi mais proeminente em pacientes com crises parciais. Os autores sugerem que o tratamento voltado para a redução dessas descargas pode contribuir para a melhora de problemas comportamentais em CCE. McLellan et al. ${ }^{(36)}$ estudaram a freqüência de distúrbios psiquiátricos antes e após tratamento cirúrgico de 60 crianças com crises focais de origem em lobo temporal. Não houve redução das comorbidades psiquiátricas após o procedimento (freqüência geral de $72 \%$, inclusive com prevalências mais elevadas de transtornos da conduta - 50\% e autismo - 38\%).

\section{Prognóstico de Saúde Mental de CCE}

Nenhum estudo foi encontrado analisando o curso das comorbidades psiquiátricas de CCE até a vida adulta. Isso provavelmente é devido ao recente interesse pelo assunto. Kokkonen ${ }^{(32)}$ avaliou 81 adultos jovens com diagnóstico de epilepsia desde a infância, comparando-os com 52 portadores de paralisia cerebral e 211 controles. Não encontrou diferença de prevalência de distúrbios psiquiátricos ativos entre os portadores de epilepsia e controles, mas sim com os portadores de paralisia cerebral. Não há referência neste estudo a diagnóstico neuropsiquiátrico no período da infância. O estudo é transversal, não sendo propriamente de prognóstico.

\section{CONCLUSÕES}

Fica claro pela exposição acima que o tratamento moderno da CCE ultrapassa o simples controle das crises epilépticas, devendo ser abrangidos o adequado diagnósti- 
co e tratamento das comorbidades psiquiátricas a fim de melhorar a qualidade de vida. Embora a prevalência dos transtornos mentais seja alta, muitos fatores afetam nossa capacidade de compreender sua real extensão e bases causais. Podemos citar as amostras heterogêneas, geralmente de natureza clínica, privilegiando epilepsias refratárias e sintomáticas; o uso de instrumentos diagnósticos diversos que podem sofrer influência das próprias crises na sintomatologia relatada e a natureza transversal da maioria dos estudos. Os futuros estudos devem privilegiar amostras mais bem definidas com análises multifatoriais e iniciar as investigações sobre a psicofarmacologia nos transtornos mentais em epilepsia infantil.

\section{REFERENNCIAS}

1. Adewuya AO Ola BA. Prevalence of and risk factors for anxiety and depressive disorders in Nigerian adolescents with epilepsy. Epilepsy Behav 2005; 6(3):342-7.

2. Adewuya AO, Oseni SBA. Impact of psychiatric morbidity on parent-rated quality of life in Nigerian adolescents with epilepsy. Epilepsy Behav 2005; 7(3):497-501.

3. Aldenkamp AP, Arends J. Effects of epileptiform EEG discharges on cognitive function: is the concept of "transient cognitive impairment” still valid? Epilepsy Behav 2004; 5 Suppl 1:S25-34.

4. Aldenkamp AP, Overweg-Plandsoen WC, Arends J. An open, nonrandomized clinical comparative study evaluating the effect of epilepsy on learning. J Child Neurol 1999; 14(12):795-800.

5. Austin JK, Dunn DW, Huster GA. Childhood epilepsy and asthma: changes in behavior problems related to gender and change in condition severity. Epilepsia 2000; 41(5):615-23.

6. Austin JK, Dunn DW. Progressive behavioral changes in children with epilepsy. Prog Brain Res 2002; 135:419-27.

7. Baki O, Erdogan A, Kantarci O, Akisik G, Kayaalp L, Yalcinkaya C. Anxiety and depression in children with epilepsy and their mothers. Epilepsy Behav 2004; 5:958-64.

8. Becker DA, Fennell EB, Carney PR. Daytime behavior and sleep disturbance in childhood epilepsy. Epilepsy Behav 2004;5:708-15.

9. Berg AT, Smith SN, Frobish D, Beckerman B, Levy SR, Testa FM, Shinnar S. Longitudinal assessment of adaptive behavior in infants and young children with newly diagnosed epilepsy: influences of etiology, syndrome, and seizure control. Pediatrics 2004; 114(3):645-50.

10. Besag FMC. Behavioral aspects of pediatric epilepsy syndromes. Epilepsy Behav 2004; 5:S3-S13.

11. Brent DA, Crumrine PK, Varma RR, Allan M, Allman C. Phenobarbital treatment and major depressive disorder in children with epilepsy. Pediatrics 1987; 80(6):909-17.

12. Brent DA, Crumrine PK, Varma R, Brown RV, Allan MJ. Phenobarbital treatment and major depressive disorder in children with epilepsy: a naturalistic follow-up. Pediatrics 1990; 85(6): 1086-91.

13. Buelow JM, Austin JK, Perkins SM, Shen J, Dunn DW, Fastenau PS. Behavior and mental health problems in children with epilepsy and low IQ. Dev Med Child Neurol 2003; 45(10):683-92.

14. Canitano R, Luchetti A, Zappella M. Epilepsy, electroencephalographic abnormalities, and regression in children with autism. J Child Neurol 2005; $20(1): 27-31$.

15. Caplan R, Arbelle S, Guthrie D, Komo S, Shields WD, Hansen R, Chayasirisobhon S. Formal thought disorder and psychopathology in pediatric primary generalized and complex partial epilepsy. J Am Acad Child Adolesc Psychiatry 1997; 36(9):1286-94.

16. Caplan R, Arbelle S, Magharious W, Guthrie D, Komo S, Shields WD, Chayasirisobhon S, Hansen R. Psychopathology in pediatric complex partial and primary generalized epilepsy. Dev Med Child Neurol 1998; 40(12):805-11.
17. Caplan R, Austin JK. Behavioral aspects of epilepsy in children with mental retardation. Ment Retard Dev Disabil Res Rev 2000; 6(4):293-9.

18. Caplan R, Siddarth P, Gurbani S, Hanson R, Sankar R, Shields WD. Depression and anxiety disorders in pediatric epilepsy. Epilepsia 2005; 46(5):720-30.

19. Carlsson G, Igelbrink-Schulze N, Neubauer BA, Stephani U. Neuropsychological long-term outcome of rolandic EEG traits. Epileptic Disord 2000; 2(Sup. 1):S63-6.

20. Cortesi F, Giannotti F, Ottaviano S. Sleep problems and daytime behavior in childhood idiopathic epilepsy. Epilepsia 1999; 40(11):1557-65.

21. Datta SS, Premkumar TS, Chandy S, Kumar S, Kirubakaran C, Gnanamuthu C, Cherian A. Behaviour problems in children and adolescents with seizure disorder: associations and risk factors. Seizure 2005; 14(3):190-7.

22. Davies S, Heyman I, Goodman R. A population survey of mental health problems in children with epilepsy. Dev Med Child Neurol 2003; 45(5):292-5.

23. Dunn DW, Austin JK, Harezlak J, Ambrosius WT. ADHD and epilepsy in childhood. Dev Med Child Neurol 2003; 45(1):50-4.

24. Dunn DW, Austin JK. Differential diagnosis and treatment of psychiatric disorders in children and adolescents with epilepsy. Epilepsy Behav 2004; 5:S10-S17.

25. Gabis L, Pomeroy J, Andriola MR. Autism and epilepsy: cause, consequence, comorbidity or coincidence? Epilepsy Behav 2005; 7:652-6.

26. Gazzaniga SM, Heartherton T.F. Ciência psicológica: mente cérebro e comportamento. Porto Alegre: Artmed; 2005. p. 413-4.

27. Gordon N. Cognitive functions and epileptic activity. Seizure 2000; 9(3):184-8.

28. Hesdorffer DC, Ludvigsson P, Olafsson E, Gudmundsson G, Kjartansson O, Hauser WA. ADHD as a risk factor for incident unprovoked seizures and epilepsy in children. Arch Gen Psychiatry 2004; 61(7):731-6.

29. Hodes M, Garralda ME, Rose G, Schwartz R. Maternal expressed emotion and adjustment in children with epilepsy. J Child Psychol Psychiatry 1999; 40(7):1083-93.

30. Hrdlicka M, Komarek V, Propper L, Kulisek R, Zumrova A, Faladova L, Havlovicova M, Sedlacek Z, Blatny M, Urbanek T. Not EEG abnormalities but epilepsy is associated with autistic regression and mental functioning in childhood autism. Eur Child Adolesc Psychiatry 2004; 13(4):209-13.

31. Keene DL, Manion I, Whiting S, Belanger E, Brennan R, Jacob P, Humphreys P. A survey of behavior problems in children with epilepsy. Epilepsy Behav 2005; 6(4):581-6.

32. Kokkonen ER, Kokkonen J, Saukkonen AL. Do neurological disorders in childhood pose a risk for mental health in young adulthood? Dev Med Child Neurol 1998; 40(6):364-8.

33. Levin B, Duchowny M. Childhood obsessive-compulsive disorder and cingulate epilepsy. Biol Psychiatry 1991; 30(10):1049-55.

34. Lewis JN, Tonge BJ, Mowat DR, Einfeld SL, Siddons HM, Rees VW. Epilepsy and associated psychopathology in young people with intellectual disability. J Paediatr Child Health 2000; 36(2): $172-5$.

35. Mangano S, Fontana A, Cusumano L. Benign myoclonic epilepsy in infancy: neuropsychological and behavioural outcome. Brain Devel 2005; 27:218-23.

36. McLellan A, Davies S, Heyman I, Harding B, Harkness W, Taylor D, Neville BG, Cross JH. Psychopathology in children with epilepsy before and after temporal lobe resection. Dev Med Child Neurol 2005; $47(10): 666-72$.

37. Monjauze C, Tuller L, Hommet C, Barthez MA, Khomsi A. Language in benign childhood epilepsy with centro-temporal spikes abbreviated form: rolandic epilepsy and language. Brain Lang 2005; 92(3):300-8.

38. Mouridsen SE, Rich B, Isager T. Epilepsy in disintegrative psychosis and infantile autism: a long-term validation study. Dev Med Child Neurol 1999; 41(2):110-4. 
39. Munoz-Yunta JA, Salvado B, Ortiz-Alonso T, Amo C, FernandezLucas A, Maestu F, Palau-Baduell M. [Clinical features of epilepsy in autism spectrum disorders. Rev Neurol. 2003; 36 Suppl 1:S61-7.

40. Noeker M, Haverkamp-Krois A, Haverkamp F. Development of mental health dysfunction in childhood epilepsy. Brain Dev. 2005; 27(1):5-16

41. Oostrom KJ, Schouten A, Kruitwagen CL, Peters AC, JennekensSchinkel A; Dutch Study Group of Epilepsy in Childhood (DuSECH). Epilepsy-related ambiguity in rating the child behavior checklist and the teacher's report form. Epileptic Disord 2001; 3(1):39-45.

42. Oostrom KJ, Schouten A, Kruitwagen CL, Peters AC, JennekensSchinkel A; Dutch Study of Epilepsy in Childhood (DuSECh). Attention deficits are not characteristic of schoolchildren with newly diagnosed idiopathic or cryptogenic epilepsy. Epilepsia 2002; 43(3):301-10.

43. Oostrom KJ, Schouten A, Olthof T, Peters AC, Jennekens-Schinkel A. Negative emotions in children with newly diagnosed epilepsy. Epilepsia. 2000; 41(3):326-31.

44. Oostrom KJ, Smeets-Schouten A, Kruitwagen CL, Peters AC, Jennekens-Schinkel A; Dutch Study Group of Epilepsy in Childhood. Not only a matter of epilepsy: early problems of cognition and behavior in children with "epilepsy only" - a prospective, longitudinal, controlled study starting at diagnosis. Pediatrics 2003; 112 (6 Pt 1):1338-44.

45. Ott D, Caplan R, Guthrie D, Siddarth P, Komo S, Shields WD, Sankar R, Kornblum H, Chayasirisobhon S. Measures of psychopathology in children with complex partial seizures and primary generalized epilepsy with absence. J Am Acad Child Adolesc Psychiatry 2001; 40(8):907-14.

46. Ott D, Siddarth P, Gurbani S, Koh S, Tournay A, Shields WD, Caplan R. Behavioral disorders in pediatric epilepsy: unmet psychiatric need. Epilepsia 2003; 44(4):591-7.

47. Papavasiliou A, Vassilaki N, Paraskevoulakos E, Kotsalis Ch, Bazigou H, Bardani I. Psychogenic status epilepticus in children. Epilepsy Behav 2004; 5(4):539-46.

48. Papavasiliou A, Mattheou D, Bazigou H, Kotsalis C, Paraskevoulakos E. Written language skills in children with benign childhood epilepsy with centrotemporal spikes. Epilepsy Behav 2005; 6(1):50-8.

49. Parkinson GM. High incidence of language disorder in children with focal epilepsies. Dev Med Child Neurol 2002; 44(8):533-7.

50. Pavone P, Incorpora G, Fiumara A, Parano E, Trifiletti RR, Ruggieri M. Epilepsy is not a prominent feature of primary autism. Neuropediatrics 2004; 35(4):207-10.
51. Pellock JM. Defining the problem: psychiatric and behavioral comorbidity in children and adolescents with epilepsy. Epilepsy Behav 2004; 5(Sup. 3):S3-9.

52. Pellock JM. Understanding co-morbidities affecting children with epilepsy. Neurology 2004; 62(5 Sup 2):S17-23.

53. Pradhan PV, Shah H, Rao P, Ashturkar D, Ghaisas P. Psychopathology and self-esteem in chronic illness. Indian J Pediatr 2003; 70(2):135-8.

54. Pressler RM, Robinson RO, Wilson GA, Binnie CD. Treatment of interictal epileptiform discharges can improve behavior in children with behavioral problems and epilepsy. J Pediatr 2005; 146(1): $112-7$.

55. Rodenburg R, Meijer AM, Dekovic M, Aldenkamp AP. Family factors and psychopathology in children with epilepsy: a literature review. Epilepsy Behav 2005; 6(4):488-503.

56. Rodenburg R, Stams GJ, Meijer AM, Aldenkamp AP, Dekovic M.J Psychopathology in children with epilepsy: a meta-analysis. Pediatr Psychol 2005; 30(6):453-68.

57. Schubert R. Attention deficit disorder and epilepsy. Pediatr Neurol 2005; $32(1): 1-10$.

58. Shalev RS. Developmental dyscalculia. J Child Neurol 2004; 19(10):765-71.

59. Steffenburg S, Gillberg C, Steffenburg U. Psychiatric disorders in children and adolescents with mental retardation and active epilepsy. Arch Neurol 1996; 53(9):904-12.

60. Steffenburg S, Steffenburg U, Gillberg C. Autism spectrum disorders in children with active epilepsy and learning disability: comorbidity, pre - and perinatal background, and seizure characteristics. Dev Med Child Neurol 2003; 45(11):724-30.

61. Thome-Souza S, Kuczynski E, Assumpção Jr. F, Rzezak P, Fuentes $\mathrm{D}$, Fiore L, Valente KD. Wich factors play a pivotal role on determining the type of psychiatric disorder in children and adolescents with epilepsy? Epilepsy Behav 2004; 5:988-94.

62. Wheless JW, Bourgeois BF. Choosing antiepileptic drugs for developmentally normal children with specific epilepsy syndromes and behavioral disorders. J Child Neurol 2004; 19 (Suppl 1): S39-48.

63. Williams J. Learning and behavior in children with epilepsy. Epilepsy Behav 2003; 4:107-11.

Endereço para correspondência:

Heber de Souza Maia Filho

Rua 16 de Março, 102/203 - Centro

CEP 25620-040, Petrópolis, RJ, Brasil

E-mail: heber@redetaho.com.br 